QUARTERLY OF APPLIED MATHEMATICS

VOLUME LXVII, NUMBER 1

MARCH 2009, PAGES 113-123

S 0033-569X(09)01114-3

Article electronically published on January 7, 2009

\title{
FRACTIONAL RADIAL DIFFUSION IN AN INFINITE MEDIUM WITH A CYLINDRICAL CAVITY
}

\author{
BY \\ Y. Z. POVSTENKO
}

Institute of Mathematics and Computer Science, Jan Dtugosz University of Czestochowa, al. Armii Krajowej 13/15, 42-200 Częstochowa, Poland

Abstract. The time-fractional diffusion equation is employed to study the radial diffusion in an unbounded body containing a cylindrical cavity. The Caputo fractional derivative is used. The solution is obtained by application of Laplace and Weber integral transforms. Several examples of problems with Dirichlet and Neumann boundary conditions are presented. Numerical results are illustrated graphically.

1. Introduction. In recent years considerable interest has been shown in the timefractional diffusion equation

$$
\frac{\partial^{\alpha} c}{\partial t^{\alpha}}=a \Delta c, \quad 0<\alpha \leq 2
$$

which is a mathematical model of a wide range of important physical phenomena (see, e.g. [1- 6] and references therein).

Two types of anomalous transport can be distinguished. The slow diffusion is exemplified by the value $0<\alpha<1$, whereas the fast diffusion is characterized by the value $1<\alpha<2$. The limiting cases $\alpha=0$ and $\alpha=2$ are known as localized diffusion and ballistic diffusion [7] $[8]$ and correspond to the Helmholtz and wave equations, respectively.

At the level of individual particle motions, the classical diffusion corresponds to the Brownian motion that is characterized by a mean-squared displacement increasing linearly with time:

$$
\left\langle x^{2}\right\rangle \sim a t .
$$

Anomalous diffusion, which is exemplified by a mean-squared displacement with the power-law time dependence,

$$
\left\langle x^{2}\right\rangle \sim a t^{\alpha}, \quad \alpha \neq 1,
$$

Received July 14, 2007.

2000 Mathematics Subject Classification. Primary 26A33.

E-mail address: j.povstenko@ajd.czest.pl 
at the level of individual particle motion, has been modeled in numerous ways. The continuous time random walk theory (see [4, 9, [10, and references therein) is more commonly used and allows one to extend classical Brownian random walks to variable jump lengths and waiting times between successive jumps. In the jump model, the particle moves instantaneously to a new site. Longer jumps are less probable. In the velocity model [11, 12 in the continuous time random walk framework, the particle moves at a constant velocity to the new site. The power-law tails make it possible to have very long waiting times, and in the case $0<\alpha<1$, particles, on average, move slower than in ordinary diffusion, whereas in the case $1<\alpha<2$, particles, on average, move faster than in ordinary diffusion.

The fundamental solution for Equation (1) in one space dimension was obtained by Mainardi [13] who also considered the signaling problem and the evolution of the initial box-signal [1] using the Laplace transform. Wyss [14] obtained the solution of the Cauchy and signaling problems in terms of $H$-functions using the Mellin transform. Schneider and Wyss [15] converted the diffusion-wave equation with appropriate initial conditions into the integrodifferential equation and found the corresponding Green functions in terms of Fox functions. Metzler and Klafter [16] considered the fractional diffusion equation on a half-line and a segment with reflecting (the Neumann problem) or absorbing (the Dirichlet problem) boundary conditions. Hilfer 17 presented a solution of the fractional diffusion equation based on Riemann-Liouville fractional derivatives in terms of $H$-functions using the Fourier, Laplace, and Mellin transforms. Hanyga [18] studied Green's functions and propagator functions in one, two, and three dimensions. Several axial-symmetric problems for a plane in cylindrical coordinates and central-symmetric problems for an infinite space in spherical coordinates were solved in [19]-[23].

In this paper we consider radial diffusion in an unbounded body containing a cylindrical cavity. The Laplace transform with respect to time and the Weber transform with respect to spatial coordinates are employed to reduce the problem to an ordinary algebraic equation. Inversion of the Laplace transform is carried out analytically in terms of Mittag-Leffler functions; inversion of the Weber transform is performed numerically.

2. Statement of the problem. We study the time-fractional radial diffusion equation

$$
\frac{\partial^{\alpha} c}{\partial t^{\alpha}}=a\left(\frac{\partial^{2} c}{\partial r^{2}}+\frac{1}{r} \frac{\partial c}{\partial r}\right), \quad 0<\alpha \leq 2,
$$

in the domain $R<r<\infty, 0<t<\infty$, under zero initial conditions:

$$
\begin{array}{ll}
t=0: & c=0, \quad 0<\alpha \leq 2, \\
t=0: & \frac{\partial c}{\partial t}=0, \quad 1<\alpha \leq 2 .
\end{array}
$$

In this paper two types of boundary conditions at the surface $r=R$ are considered: the Dirichlet boundary condition with the prescribed boundary value of function

$$
r=R: \quad c=u(t),
$$


and the Neumann boundary condition with the prescribed boundary value of normal derivative

$$
r=R: \quad \frac{\partial c}{\partial r}=w(t) .
$$

The zero condition at infinity is also assumed:

$$
\lim _{r \rightarrow \infty} c(r, t)=0 .
$$

In Equation (4), the Caputo fractional derivative of the order $\alpha$ is used. Below we briefly recall the main ideas of fractional calculus 24, 25. Integrating by parts $n-1$ times, the calculation of the $n$-fold primitive of a function $f(t)$ can be reduced to the calculation of a single integral of the convolution type

$$
I^{n} f(t)=\frac{1}{(n-1) !} \int_{0}^{t}(t-\tau)^{n-1} f(\tau) \mathrm{d} \tau,
$$

where $n$ is a positive integer. The Riemann-Liouville fractional integral is introduced as a natural generalization of the convolution type form (10):

$$
I^{\alpha} f(t)=\frac{1}{\Gamma(\alpha)} \int_{0}^{t}(t-\tau)^{\alpha-1} f(\tau) \mathrm{d} \tau, \quad \alpha>0,
$$

where $\Gamma(\alpha)$ is the gamma function.

The Riemann-Liouville fractional derivative is defined as left-inverse to $I^{\alpha}$ :

$$
\frac{\mathrm{d}_{\mathrm{RL}}^{\alpha} f(t)}{\mathrm{d} t^{\alpha}}= \begin{cases}\frac{\mathrm{d}^{n}}{\mathrm{~d} t^{n}}\left[\frac{1}{\Gamma(n-\alpha)} \int_{0}^{t}(t-\tau)^{n-\alpha-1} f(\tau) \mathrm{d} \tau\right], & n-1<\alpha<n, \\ \frac{\mathrm{d}^{n} f(t)}{\mathrm{d} t^{n}}, & \alpha=n,\end{cases}
$$

and its Laplace transform rule requires knowledge of the initial values of the fractional integral $I^{n-\alpha} f(t)$ and its derivatives of the order $k=1,2, \ldots, n-1$ :

$$
\mathcal{L}\left\{\frac{\mathrm{d}_{\mathrm{RL}}^{\alpha} f(t)}{\mathrm{d} t^{\alpha}}\right\}=s^{\alpha} \mathcal{L}\{f(t)\}-\sum_{k=0}^{n-1} \frac{\mathrm{d}^{k}}{\mathrm{~d} t^{k}} I^{n-\alpha} f\left(0^{+}\right) s^{n-1-k}, \quad n-1<\alpha<n,
$$

where $s$ is the transform variable.

The Caputo derivative of the fractional order $\alpha$ is defined as

$$
\frac{\mathrm{d}^{\alpha} f(t)}{\mathrm{d} t^{\alpha}}= \begin{cases}\frac{1}{\Gamma(n-\alpha)} \int_{0}^{t}(t-\tau)^{n-\alpha-1} \frac{\mathrm{d}^{n} f(\tau)}{\mathrm{d} \tau^{n}} \mathrm{~d} \tau, & n-1<\alpha<n, \\ \frac{\mathrm{d}^{n} f(t)}{\mathrm{d} t^{n}}, & \alpha=n,\end{cases}
$$

and its Laplace transform rule requires knowledge of the initial values of the function $f(t)$ and its integer derivatives of order $k=1,2, \ldots, n-1$ :

$$
\mathcal{L}\left\{\frac{\mathrm{d}^{\alpha} f(t)}{\mathrm{d} t^{\alpha}}\right\}=s^{\alpha} \mathcal{L}\{f(t)\}-\sum_{k=0}^{n-1} f^{(k)}\left(0^{+}\right) s^{\alpha-1-k}, \quad n-1<\alpha<n .
$$

These two types of fractional derivatives are connected by the following relation [24]:

$$
\frac{\mathrm{d}_{\mathrm{RL}}^{\alpha}}{\mathrm{d} t^{\alpha}}=\frac{\mathrm{d}^{\alpha} f(t)}{\mathrm{d} t^{\alpha}}+\sum_{k=0}^{n-1} \frac{t^{k-\alpha}}{\Gamma(k-\alpha+1)} f^{(k)}\left(0^{+}\right), \quad n-1<\alpha<n, \quad t>0,
$$


and coincide under condition that the function $f(t)$ along with its first $n-1$ derivatives vanishes at $t=0^{+}$. If care is taken, the results obtained using the Caputo formulation can be recast to the Riemann-Liouville version.

To solve problems in an unbounded medium with cylindrical cavity, the Weber integral transform with respect to spatial coordinate $r$ is of great significance. As the Weber transform is less known than the usual Hankel transform, we recall its properties in the Appendix.

3. The Dirichlet boundary condition. Consider the initial-boundary-value problem (4)-(7) with constant boundary value specification

$$
r=R: \quad c=u_{0}=\text { const. }
$$

Applying the Laplace integral transform with respect to time $t$ (denoted by the asterisk) and the Weber transform with respect to coordinate $r$ (denoted by the overbar) and taking into account formulae (15) and (A.9), we obtain

$$
\bar{c}^{*}=-\frac{2 a u_{0}}{\pi} \frac{1}{\sqrt{J_{0}^{2}(R \xi)+Y_{0}^{2}(R \xi)}} \frac{1}{s\left(s^{\alpha}+a \xi^{2}\right)},
$$

where $s$ is the Laplace transform variable and $\xi$ is the Weber transform variable.

Carrying out the inverse integral transforms gives

$$
c=\frac{2 u_{0}}{\pi} \int_{0}^{\infty}\left[E_{\alpha}\left(-a \xi^{2} t^{\alpha}\right)-1\right] \frac{J_{0}(r \xi) Y_{0}(R \xi)-Y_{0}(r \xi) J_{0}(R \xi)}{J_{0}^{2}(R \xi)+Y_{0}^{2}(R \xi)} \frac{\mathrm{d} \xi}{\xi},
$$

where

$$
E_{\alpha}(z)=\sum_{n=0}^{\infty} \frac{z^{n}}{\Gamma(\alpha n+1)}, \quad \alpha>0, z \in C,
$$

is the Mittag-Leffler function [26] providing a generalization of the exponential function. It is evident that for $x>0$,

$$
E_{1}(-x)=\mathrm{e}^{-x} \text { and } E_{2}(-x)=\cos \sqrt{x} .
$$

The Mittag-Leffler function $E_{\alpha}(-x)$ has the following asymptotic behavior as $x \rightarrow \infty$ 24:

$$
E_{\alpha}(-x) \sim \frac{1}{\Gamma(1-\alpha) x}, \quad x \rightarrow \infty, \quad 0<\alpha<2, \quad \alpha \neq 1 .
$$

Using the integral (A.14) and introducing the dimensionless parameters $\eta=R \xi, \rho=$ $r / R, \kappa=\sqrt{a} t^{\alpha / 2} / R$, Equation (19) can be rewritten as

$$
\frac{c}{u_{0}}=1+\frac{2}{\pi} \int_{0}^{\infty} E_{\alpha}\left(-\kappa^{2} \eta^{2}\right) \frac{J_{0}(\rho \eta) Y_{0}(\eta)-Y_{0}(\rho \eta) J_{0}(\eta)}{J_{0}^{2}(\eta)+Y_{0}^{2}(\eta)} \frac{\mathrm{d} \eta}{\eta} .
$$

Let us consider several particular cases. The solution of the classical diffusion equation is obtained when $\alpha=1$ and is well known 27, 28:

$$
\frac{c}{u_{0}}=1+\frac{2}{\pi} \int_{0}^{\infty} \exp \left(-\kappa^{2} \eta^{2}\right) \frac{J_{0}(\rho \eta) Y_{0}(\eta)-Y_{0}(\rho \eta) J_{0}(\eta)}{J_{0}^{2}(\eta)+Y_{0}^{2}(\eta)} \frac{\mathrm{d} \eta}{\eta} .
$$

The limiting case $\alpha \rightarrow 0$ corresponds to the solution of the Helmholtz equation:

$$
\frac{c}{u_{0}}=1+\frac{2}{\pi} \int_{0}^{\infty} \frac{1}{1+\kappa^{2} \eta^{2}} \frac{J_{0}(\rho \eta) Y_{0}(\eta)-Y_{0}(\rho \eta) J_{0}(\eta)}{J_{0}^{2}(\eta)+Y_{0}^{2}(\eta)} \frac{\mathrm{d} \eta}{\eta},
$$




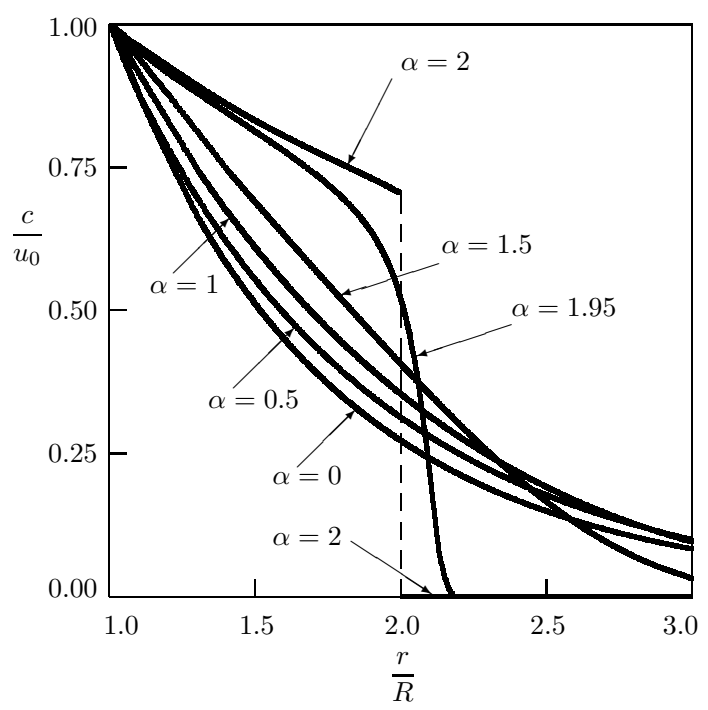

Figure 1. Dependence of concentration on distance (the Dirichlet problem with constant boundary condition; $\kappa=1$ ).

while the limiting case $\alpha=2$ corresponds to the solution of the wave equation:

$$
\frac{c}{u_{0}}=1+\frac{2}{\pi} \int_{0}^{\infty} \cos (\kappa \eta) \frac{J_{0}(\rho \eta) Y_{0}(\eta)-Y_{0}(\rho \eta) J_{0}(\eta)}{J_{0}^{2}(\eta)+Y_{0}^{2}(\eta)} \frac{\mathrm{d} \eta}{\eta}
$$

The results of numerical calculations based on Equations (23)-(26) are presented in Figure 1.

Consider next the instantaneous delta-pulse at the boundary:

$$
r=R: \quad c=U_{0} \delta_{+}(t) .
$$

In this case

$$
\bar{c}^{*}=-\frac{2 a U_{0}}{\pi} \frac{1}{\sqrt{J_{0}^{2}(R \xi)+Y_{0}^{2}(R \xi)}} \frac{1}{s^{\alpha}+a \xi^{2}}
$$

and

$$
\frac{c t}{U_{0}}=-\frac{2 \kappa^{2}}{\pi} \int_{0}^{\infty} E_{\alpha, \alpha}\left(-\kappa^{2} \eta^{2}\right) \frac{J_{0}(\rho \eta) Y_{0}(\eta)-Y_{0}(\rho \eta) J_{0}(\eta)}{J_{0}^{2}(\eta)+Y_{0}^{2}(\eta)} \eta \mathrm{d} \eta
$$

where $E_{\alpha, \beta}(z)$ is the generalized Mittag-Leffler function in two parameters [24, 25]:

$$
E_{\alpha, \beta}(z)=\sum_{n=0}^{\infty} \frac{z^{n}}{\Gamma(\alpha n+\beta)}, \quad \alpha>0, \beta>0, z \in C .
$$

Simple special cases of $E_{\alpha, \beta}(z)$ are

$$
E_{1,1}(-x)=\mathrm{e}^{-x} \quad \text { and } \quad E_{2,2}(-x)=\frac{\sin \sqrt{x}}{\sqrt{x}} .
$$

The behavior of the generalized Mittag-Leffler function $E_{\alpha, \alpha}(-x)$ as $x \rightarrow \infty$ is described by asymptotic representation:

$$
E_{\alpha, \alpha}(-x) \sim-\frac{1}{\Gamma(-\alpha) x^{2}}, \quad x \rightarrow \infty, \quad 0<\alpha<2, \quad \alpha \neq 1 .
$$




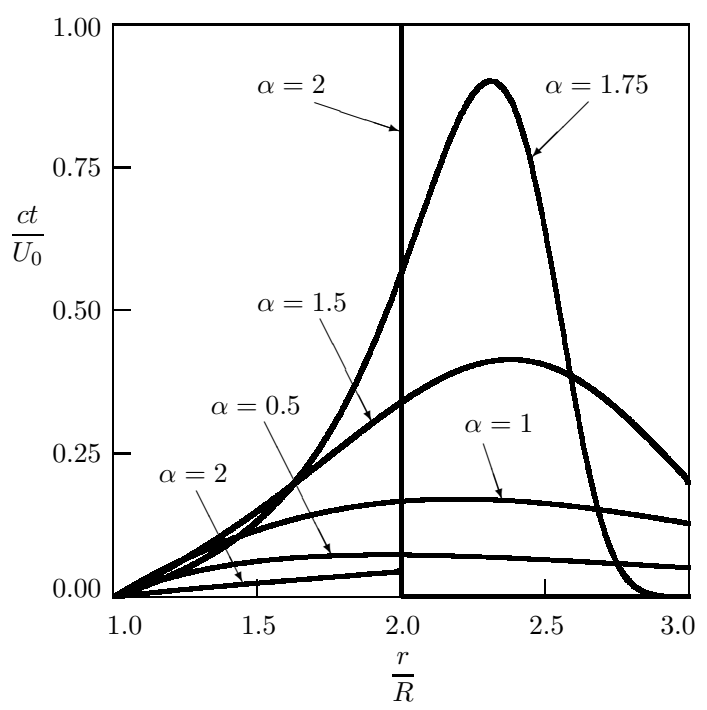

Figure 2. Dependence of concentration on distance (the Dirichlet problem with Dirac's delta boundary condition; $\kappa=1$ ).

Two particular cases of solution (29) can be examined: the solution of classical diffusion equation $(\alpha=1)$ :

$$
\frac{c t}{U_{0}}=-\frac{2 \kappa^{2}}{\pi} \int_{0}^{\infty} \exp \left(-\kappa^{2} \eta^{2}\right) \frac{J_{0}(\rho \eta) Y_{0}(\eta)-Y_{0}(\rho \eta) J_{0}(\eta)}{J_{0}^{2}(\eta)+Y_{0}^{2}(\eta)} \eta \mathrm{d} \eta
$$

and the wave equation $(\alpha=2)$ :

$$
\frac{c t}{U_{0}}=-\frac{2 \kappa}{\pi} \int_{0}^{\infty} \sin (\kappa \eta) \frac{J_{0}(\rho \eta) Y_{0}(\eta)-Y_{0}(\rho \eta) J_{0}(\eta)}{J_{0}^{2}(\eta)+Y_{0}^{2}(\eta)} \mathrm{d} \eta .
$$

Figure 2 shows the variation of concentration with distance according to Equations (29), (33), and (34). The vertical line at $\rho=2$ represents Dirac's delta $\frac{1}{\sqrt{1+\kappa}} \delta(\rho-1-\kappa)$ for $\kappa=1$.

4. The Neumann boundary condition. In this section the initial-boundary-value problem (4)-(6), (8) is treated under specified normal derivative

$$
r=R: \quad \frac{\partial c}{\partial r}=-w_{0}=\text { const. }
$$

The Laplace and Weber transform techniques and taking into account (15) and (A.13) results in the following equation:

$$
\bar{c}^{*}=\frac{2 a w_{0}}{\pi} \frac{1}{\sqrt{J_{1}^{2}(R \xi)+Y_{1}^{2}(R \xi)}} \frac{1}{s\left(s^{\alpha}+a \xi^{2}\right)},
$$

or, after inverting the integral transforms,

$$
\frac{c}{R w_{0}}=\frac{2}{\pi} \int_{0}^{\infty}\left[E_{\alpha}\left(-\kappa^{2} \eta^{2}\right)-1\right] \frac{J_{0}(\rho \eta) Y_{1}(\eta)-Y_{0}(\rho \eta) J_{1}(\eta)}{J_{1}^{2}(\eta)+Y_{1}^{2}(\eta)} \frac{\mathrm{d} \eta}{\eta^{2}},
$$




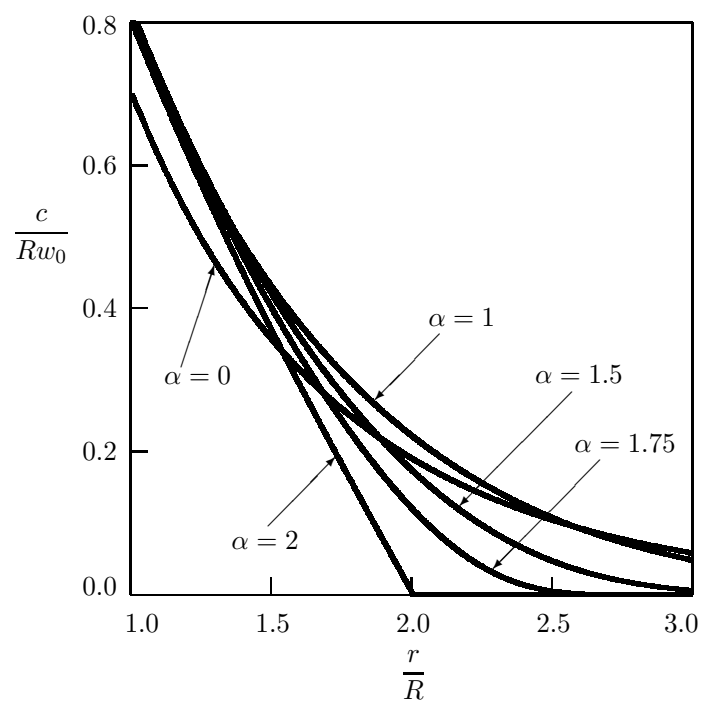

Figure 3. Dependence of concentration on distance (the Neumann problem with constant boundary condition; $\kappa=1$ ).

with particular cases corresponding to the standard diffusion equation $(\alpha=1)$ with $E_{\alpha}\left(-\kappa^{2} \eta^{2}\right)$ replaced by $\exp \left(-\kappa^{2} \eta^{2}\right)$ (see [27, 28), to the Helmholtz equation $(\alpha \rightarrow 0)$ with $E_{\alpha}\left(-\kappa^{2} \eta^{2}\right)$ replaced by $\frac{1}{1+\kappa^{2} \eta^{2}}$, and to the wave equation $(\alpha=2)$ with $\cos (\kappa \eta)$ replaced by $E_{\alpha}\left(-\kappa^{2} \eta^{2}\right)$.

Figure 3 illustrates the dependence of concentration on distance for various values of $\alpha$.

In the case of instantaneous delta-pulse specification of normal derivative,

$$
r=R: \quad \frac{\partial c}{\partial r}=-W_{0} \delta_{+}(t)
$$

we get

$$
\frac{c t}{R W_{0}}=-\frac{2 \kappa^{2}}{\pi} \int_{0}^{\infty} E_{\alpha, \alpha}\left(-\kappa^{2} \eta^{2}\right) \frac{J_{0}(\rho \eta) Y_{1}(\eta)-Y_{0}(\rho \eta) J_{1}(\eta)}{J_{1}^{2}(\eta)+Y_{1}^{2}(\eta)} \mathrm{d} \eta .
$$

Particular cases of Equation (39) correspond to the standard diffusion equation $(\alpha=1)$ and the wave equation $(\alpha=2)$ and are obtained replacing $E_{\alpha, \alpha}\left(-\kappa^{2} \eta^{2}\right)$ with $\exp \left(-\kappa^{2} \eta^{2}\right)$ and $\frac{\sin (\kappa \eta)}{\kappa \eta}$, respectively.

The results of numerical calculations are displayed in Figure 4.

5. Discussion and conclusions. The results given by Equations (23), (29), (37), and (39) and displayed in Figures 1-4 are the primary results of this paper. The solutions of time-fractional diffusion equations satisfy the appropriate boundary conditions at the surface $r=R$ and reduce to the solutions of classical diffusion equations in the limit $\alpha=1$. In the case where $0<\alpha<1$, the time-fractional diffusion equation interpolates the Helmholtz equation and the diffusion equation. In this case, when it is possible to consider the limit $\alpha \rightarrow 0$, the obtained solutions reduce to the solutions of the Helmholtz equation. In the case where $1<\alpha<2$, the time-fractional diffusion equation interpolates 


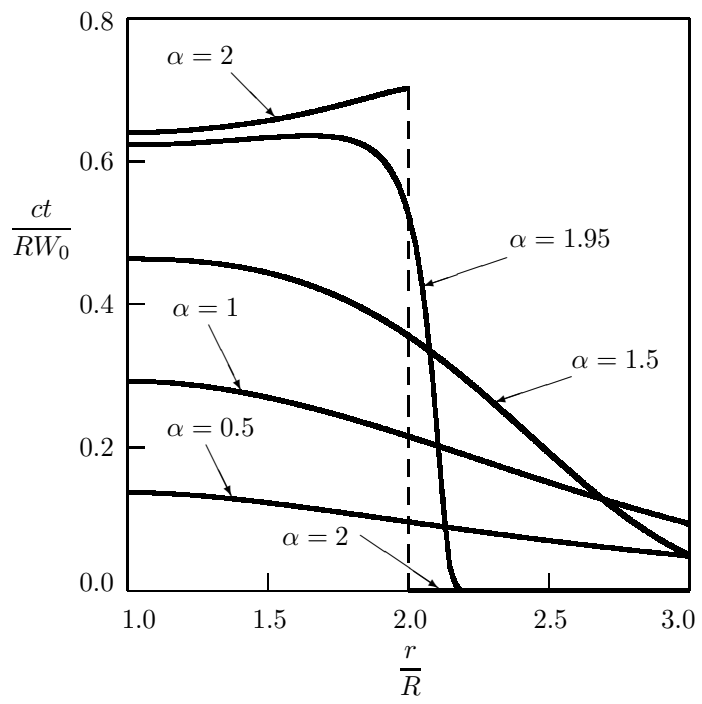

Figure 4. Dependence of concentration on distance (the Neumann problem with Dirac's delta boundary condition; $\kappa=1$ ).

the diffusion equation and the wave equation. In the limit $\alpha=2$ the obtained solutions reduce to the solutions of the wave equation. The solutions of the fractional diffusion equation in the fast diffusion regime approximate the propagating steps and humps typical for the standard wave equation in contrast to the shape of curves describing the slow diffusion regime. In particular, it is evident from the figures how wave fronts at $\rho=1+\kappa$ (the jog in Figure 3, jumps in Figures 1 and 4, and Dirac's delta in Figure 2) that arise in the case of the wave equation are approximated by the solutions of the time-fractional diffusion equation with $\alpha$ approaching 2 .

The plot of the solution for $\alpha=2$ in Figure 2 needs additional discussion. If we consider the axisymmetric Cauchy problem for the wave equation in a plane with initial value $c(r, 0)=\delta(r-R)$, then the nondimensional solution for $0<\kappa<1$ has the following form (see Equation (99) in [21]):

$$
\begin{aligned}
c & =\frac{1}{2 \sqrt{1-\kappa}} \delta(\rho-1+\kappa)+\frac{1}{2 \sqrt{1+\kappa}} \delta(\rho-1-\kappa) \\
+ & 0 \leq \begin{array}{ll}
0, & 1-\kappa<\rho<1+\kappa, \\
\frac{\kappa}{4 \pi \rho^{3 / 2}} \frac{\mathbf{E}(k)-k^{\prime 2} \mathbf{K}(k)}{k^{2} k^{\prime 2}}, & 1+\kappa<\rho<\infty, \\
0, &
\end{array}
\end{aligned}
$$

where $\mathbf{K}(k)$ and $\mathbf{E}(k)$ are the complete elliptic integrals of the first and second kind, $k=\sqrt{\kappa^{2}-(\rho-1)^{2}} /(2 \sqrt{\rho}), k^{\prime}=\sqrt{1-k^{2}}$.

The first term in Equation (40) presents the delta peak traveling in the direction of origin; the second term corresponds to the delta peak propagating in the direction of infinity; and the third term describes a "tail" behind the wave fronts. In the case of the cylinder with radius $R(0 \leq r \leq R)$ considered in 23 , the signaling problem for the wave equation with the Dirac delta boundary condition $c(R, t)=\delta_{+}(t)$ in the case 
$0<\kappa<1$ has the solution containing the delta peak traveling in the direction of origin and a portion of "tail" behind the wave front:

$$
c=\frac{1}{\sqrt{1-\kappa}} \delta(\rho-1+\kappa)+(\text { a "tail"). }
$$

Similarly, in the case of the infinite medium with a cylindrical cavity $(R \leq r<\infty)$ considered in the present paper, the corresponding solution to the signaling problem contains the delta peak traveling in the direction of infinity and also has a portion of a "tail" behind the wave front:

$$
c=\frac{1}{\sqrt{1+\kappa}} \delta(\rho-1-\kappa)+(\text { a "tail" }) .
$$

It should be noted that the coefficients of delta functions in (41) and (42) are twice as large as those in (40) (the initial delta pulse does not split into two parts).

The "tails" in (41) and (42) cannot be calculated analytically as in (40), but can be estimated numerically.

Appendix. The Weber integral transform of order $\nu$ is defined as $([28,29])$

$$
\mathcal{W}_{\nu}\{f(r)\}=\bar{f}(\xi)=\int_{R}^{\infty} K_{\nu}(r, R, \xi) f(r) r \mathrm{~d} r
$$

having the inverse

$$
\mathcal{W}_{\nu}^{-1}\{\bar{f}(\xi)\}=f(r)=\int_{0}^{\infty} K_{\nu}(r, R, \xi) \bar{f}(\xi) \xi \mathrm{d} \xi .
$$

The significance of the Weber transform for problems in polar coordinates in the domain $R<r<\infty$ is due to the following formula:

$\mathcal{W}_{\nu}\left\{\frac{\mathrm{d}^{2} f}{\mathrm{~d} r^{2}}+\frac{1}{r} \frac{\mathrm{d} f}{\mathrm{~d} r}-\frac{\nu^{2}}{r^{2}} f\right\}=-\xi^{2} \bar{f}(\xi)+\left.R f(R) \frac{\partial K_{\nu}(r, R, \xi)}{\partial r}\right|_{r=R}-\left.R K_{\nu}(R, R, \xi) \frac{\mathrm{d} f(r)}{\mathrm{d} r}\right|_{\substack{r=R \\ \text {. }}}$.

The specific expression of the kernel $K_{\nu}(r, R, \xi)$ depends on the boundary conditions at $r=R$. For the Dirichlet boundary condition, the kernel is chosen as

$$
K_{\nu}^{(D)}(r, R, \xi)=\frac{J_{\nu}(r \xi) Y_{\nu}(R \xi)-Y_{\nu}(r \xi) J_{\nu}(R \xi)}{\sqrt{J_{\nu}^{2}(R \xi)+Y_{\nu}^{2}(R \xi)}},
$$

whereas for the Neumann boundary condition,

$$
K_{\nu}^{(N)}(r, R, \xi)=\frac{J_{\nu}(r \xi) Y_{\nu}^{\prime}(R \xi)-Y_{\nu}(r \xi) J_{\nu}^{\prime}(R \xi)}{\sqrt{\left[J_{\nu}^{\prime}(R \xi)\right]^{2}+\left[Y_{\nu}^{\prime}(R \xi)\right]^{2}}},
$$

where $J_{\nu}(r)$ and $Y_{\nu}(r)$ are the Bessel functions of the first and second kind, respectively.

Formulae above considerably simplify in the case $\nu=0$ :

$$
\begin{aligned}
& K_{0}^{(D)}(r, R, \xi)=\frac{J_{0}(r \xi) Y_{0}(R \xi)-Y_{0}(r \xi) J_{0}(R \xi)}{\sqrt{J_{0}^{2}(R \xi)+Y_{0}^{2}(R \xi)}}, \\
& K_{0}^{(D)}(R, R, \xi)=0, \\
& \left.\frac{\partial K_{0}^{(D)}(r, R, \xi)}{\partial r}\right|_{r=R}=-\frac{2}{\pi R} \frac{1}{\sqrt{J_{0}^{2}(R \xi)+Y_{0}^{2}(R \xi)}},
\end{aligned}
$$




$$
\mathcal{W}_{0}^{(D)}\left\{\frac{\mathrm{d}^{2} f}{\mathrm{~d} r^{2}}+\frac{1}{r} \frac{\mathrm{d} f}{\mathrm{~d} r}\right\}=-\xi^{2} \bar{f}(\xi)-\frac{2}{\pi} \frac{1}{\sqrt{J_{0}^{2}(R \xi)+Y_{0}^{2}(R \xi)}} f(R)
$$

and

$$
\begin{aligned}
& K_{0}^{(N)}(r, R, \xi)=-\frac{J_{0}(r \xi) Y_{1}(R \xi)-Y_{0}(r \xi) J_{1}(R \xi)}{\sqrt{J_{1}^{2}(R \xi)+Y_{1}^{2}(R \xi)}}, \\
& K_{0}^{(N)}(R, R, \xi)=\frac{2}{\pi R \xi} \frac{1}{\sqrt{J_{1}^{2}(R \xi)+Y_{1}^{2}(R \xi)}}, \\
& \left.\frac{\partial K_{0}^{(N)}(r, R, \xi)}{\partial r}\right|_{r=R}=0, \\
& \mathcal{W}_{0}^{(N)}\left\{\frac{\mathrm{d}^{2} f}{\mathrm{~d} r^{2}}+\frac{1}{r} \frac{\mathrm{d} f}{\mathrm{~d} r}\right\}=-\xi^{2} \bar{f}(\xi)-\left.\frac{2}{\pi \xi} \frac{1}{\sqrt{J_{1}^{2}(R \xi)+Y_{1}^{2}(R \xi)}} \frac{\mathrm{d} f(r)}{\mathrm{d} r}\right|_{r=R} .
\end{aligned}
$$

The following integral ([30]) is used to convert Equation (19) to Equation (23):

$$
\int_{0}^{\infty} \frac{J_{\nu}(r \xi) Y_{\nu}(R \xi)-Y_{\nu}(r \xi) J_{\nu}(R \xi)}{J_{\nu}^{2}(R \xi)+Y_{\nu}^{2}(R \xi)} \frac{\mathrm{d} \xi}{\xi}=-\frac{\pi}{2}\left(\frac{R}{r}\right)^{\nu}, \quad R<r<\infty .
$$

Acknowledgement. The author would like to thank the referee for useful comments that improved the final version of the paper.

\section{REFERENCES}

[1] F. Mainardi, Fractional relaxation-oscillation and fractional diffusion-wave phenomena, Chaos, Solitons and Fractals 7 (1996), 1461-1477. MR1409912 (97i:26011)

[2] F. Mainardi, Fractional calculus: Some basic problems in continuum and statistical mechanics. In: A. Carpinteri and F. Mainardi (Eds.), Fractals and Fractional Calculus in Continuum Mechanics, Springer, Wien, 1997, 291-348. MR1611587 (99f:26010)

[3] T. F. Nonnenmacher and R. Metzler, Applications of fractional calculus techniques to problems in biophysics. In: R. Hilfer (Ed.), Applications of Fractional Calculus in Physics, World Scientific, Singapore, 2000, 377-427. MR.1890112 (2003b:26006)

[4] R. Metzler and J. Klafter, The random walk's guide to anomalous diffusion: A fractional dynamics approach, Phys. Rep. 339 (2000), 1-77. MR1809268 (2001k:82082)

[5] R. Metzler and T. F. Nonnenmacher, Space- and time-fractional diffusion and wave equations, fractional Fokker-Planck equations, and physical motivation, Chem. Phys. 284 (2002), 67-90.

[6] G. M. Zaslavsky, Chaos, fractional kinetics, and anomalous transport, Phys. Rep. 371 (2002), 461-580. MR 1937584(2003i:70030)

[7] R. Metzler and J. Klafter, Accelerated Brownian motion: A fractional dynamics approach to fast diffusion, Europhys. Lett. 51 (2000), 492-498.

[8] R. Kimmich, Strange kinetics, porous media, and NMR, Chem. Phys. 284 (2002), 43-85.

[9] E. W. Montroll and M. F. Shlesinger, On the wonderful world of random walks. In: J. L. Lebowitz and E. W. Montroll (Eds.), Nonequilibrium Phenomena II: From Stochastics to Hydrodynamics, North-Holland, Amsterdam, The Netherlands, 1984, 1-121. MR757002 (86g:82002)

[10] R. Metzler, J. Klafter, and I. M. Sokolov, Anomalous transport in external fields: Continuous time random walks and fractional diffusion equation extended, Phys. Rev. E 58 (1998), 1621-1633.

[11] G. Zumofen and J. Klafter, Scale-invariant motion in intermittent chaotic systems, Phys. Rev. E 47 (1993), 851-863.

[12] R. Metzler and A. Compte, Stochastic foundation of normal and anomalous Cattaneo-type transport, Physica A 268 (1999), 454-468.

[13] F. Mainardi, The fundamental solutions for the fractional diffusion-wave equation, Appl. Math. Lett. 9 (1996), 23-28. MR.1419811 (97h:35132) 
[14] W. Wyss, The fractional diffusion equation, J. Math. Phys. 27 (1986), 2782-2785. MR.861345 $(87 \mathrm{~m}: 44008)$

[15] W. R. Schneider and W. Wyss, Fractional diffusion and wave equations, J. Math. Phys. 30 (1989), 134-144. MR974464 (89m:45017)

[16] R. Metzler and J. Klafter, Boundary value problems for fractional diffusion equations, Physica A 278 (2000), 107-125. MR.1763650 (2001b:35138)

[17] R. Hilfer, Fractional diffusion based on Riemann-Liouville fractional derivatives, J. Phys. Chem. B 104 (2000), 3914-3917.

[18] A. Hanyga, Multidimensional solutions of time-fractional diffusion-wave equations, Proc. Roy. Soc. London A 458 (2002), 933-957. MR1898095 (2003e:35039)

[19] Y. Z. Povstenko, Fractional heat conduction equation and associated thermal stress, J. Thermal Stresses 28 (2005), 83-102. MR2119353(2005i:74024)

[20] Y. Z. Povstenko, Stresses exerted by a source of diffusion in a case of a non-parabolic diffusion equation, Int. J. Engng. Sci. 43, (2005), 977-991. MR2163182(2006c:35158)

[21] Y. Z. Povstenko, Two-dimensional axisymmetric stresses exerted by instantaneous pulses and sources of diffusion in an infinite space in a case of time-fractional diffusion equations, Int. J. Solids Struct. 44 (2007), 2324-2348. MR2295355 (2007j:74024)

[22] Y. Z. Povstenko, Fundamental solutions to three-dimensional diffusion-wave equations and associated diffusive stresses, Chaos Solitons Fractals 36 (2008), 961-972. MR2379364

[23] Y. Z. Povstenko, Fractional radial diffusion in a cylinder, J. Mol. Liq. 137 (2008), 46-50.

[24] R. Gorenflo and F. Mainardi, Fractional calculus: Integral and differential equations of fractional order, In: A. Carpinteri and F. Mainardi (Eds.), Fractals and Fractional Calculus in Continuum Mechanics, Springer, Wien, 1997, 223-276. MR.1611585 (99g:26015)

[25] A. A. Kilbas, H. M. Srivastava, and J. J. Trujillo, Theory and Applications of Fractional Differential Equations, Elsevier, Amsterdam, The Netherlands, 2006. MR2218073 (2007a:34002)

[26] A. Erdélyi, W. Magnus, F. Oberhettinger, and F. Tricomi, Higher Transcendental Functions, vol. 3, McGraw-Hill, New York, 1955. MR0066496 (16:586c)

[27] H. S. Carslaw and J. C. Jaeger, Conduction of Heat in Solids, 2nd ed., Oxford University Press, 1959. MR0022294 (9:188a)

[28] A. S. Galitsyn and A. N. Zhukovsky, Integral Transforms and Special Functions in Heat Conduction Problems, Naukova Dumka, Kiev, 1976. (In Russian).

[29] E. C. Titchmarsh, Eigenfunction Expansion Associated with Second-Order Differential Equations, Clarendon Press, Oxford, UK, 1946. MR0019765 (8:458d)

[30] A. P. Prudnikov, Y. A. Brychkov, and O. I. Marichev, Integrals and Series: Special Functions. Nauka, Mocsow, 1983. (In Russian). MR737562 (85b:33002) 